\title{
Numerical Simulation of the Sound Generation and the Sound Propagation from Air Intakes in an Aircraft Cabin
}

\author{
Mikhail Konstantinov ${ }^{1}$, Claus Wagner ${ }^{1,2}$ \\ ${ }^{1}$ German Aerospace Center, Institute of Aerodynamics and Flow Technology, SCART, \\ Bunsenstraße 10, 37073 Göttingen, Germany \\ ${ }^{2}$ Technische Universität Ilmenau, Institute of Thermodynamics and Fluid Mechanics, \\ Am Helmholtzring 1, 98693 Ilmenau, Germany \\ mikhail.konstantinov@dlr.de
}

\begin{abstract}
The paper presents results of unsteady Reynolds-averaged NavierStokes simulations (URANS), Delayed Detached Eddy Simulations (DDES) and Large Eddy Simulations (LES) of the flow and the noise propagation in a segment of the DLR's cabin test facility Do728. Since the weakly compressible Navier-Stokes equations were solved in all cases, spectra of the sound pressure levels (SPL) were analyzed based on Fast Fourier Transforms of the predicted pressure fields. It was shown that LES on hexahedral meshes predict SPLs in good agreement with microphone measurements in the Do728 cabin. A comparison with predictions based on a hybrid approach involving the solution of a wave equation together with non-reflective boundary conditions on the one hand, and a Ffowcs Williams - Hawkings (FW-H) model on the other hand, revealed that the impact of the imperfect acoustic boundary condition in the LES on the sound pressure level at the receiver point is negligible. Thus, it was demonstrated that reliable predictions of SPLs in an aircraft cabin are possible if the corresponding LES can be performed.
\end{abstract}

Keywords: URANS, DDES, LES, acoustic analogies, cabin air intake.

\section{Introduction}

In the last decade, aero-acoustical simulations for heating, ventilation and airconditioning (HVAC) systems, in particular of passenger cars, were performed in many studies, for example in [1, 4, 6, 12]. These calculations were carried out using acoustic analogies by coupling results obtained with Computational Fluid Dynamics (CFD) software packages like Star-CD [1], ANSYS [4] and OpenFOAM with acoustic models provided by Virtual.Lab and ACTRAN et al (benchmark study see in [2]). These studies have in common that they only consider noise sources located inside air intakes and the propagation into free-space. Similarly, the generation of the aerodynamic noise in- and outside a simple model of a component of an aircraft air distribu- 
tion system is predicted in [11]. Furthermore, the sound generation in a single isolated air cabin intake and the sound propagation in free space are studied in [6] using StarCCM+ on the one hand, and by coupling OpenFOAM with the software Virtual.Lab on the other hand. One main result was that the cabin walls and the location of the cabin air intakes (CAI) significantly influence the sound propagation within the cabin.

However, predicting sound pressure levels in a realistic and complete aircraft cabin is still too challenging. One reason is that there are three major aerodynamic noise sources contributing to the overall sound level in the cabin. Besides the abovementioned noise generated in the air distribution system, i.e. mainly in the CAI, engine and boundary layer noise is also transmitted from outside into the cabin. In the present study, the focus lies on the noise propagation within a cabin model originating from noise sources located in the CAI. The objective is to evaluate different approaches provided in the commercial software package StarCCM+ regarding their suitability for predicting sound pressure levels in the vicinity of passengers. In this respect, we compare solutions of different versions of unsteady weakly compressible Navier-Stokes equations in terms of the directly predicted sound pressure levels both with each other and with solutions obtained with the Ffowcs Williams - Hawkings $(\mathrm{FW}-\mathrm{H})$ model and a wave equation. The considered computational domain is a segment of the Do728 aircraft cabin with realistic CAI. It must be noted, that the NavierStokes equations are solved with Neumann boundary conditions for all flow variables (velocity and pressure) at the side boundaries of the Do728 segment. This is a common boundary condition in Computational Fluid Dynamics (CFD). However, for a wave approaching the side boundaries, these Neumann conditions will be reflective at least to some extent. Thus, the question is how these Neumann boundary conditions affect the predicted sound pressure level in the center of the cabin where passengers are seated and whether this problem can be omitted by solving a wave equation together with non-reflective boundary conditions or by using a Ffowcs Williams Hawkings (FW-H) model. The latter propagates the sound generated at all solid walls, i.e. the inner boundaries of the CAI and at all cabin walls except the side boundaries, based on pressure fluctuations which are predicted in Large Eddy Simulation (LES), Delayed Detached Eddy Simulation (DDES) or unsteady Reynolds-averaged NavierStokes Simulations (URANS) as an input. In this respect, the sound pressure levels predicted with LES, DDES and URANS are compared and evaluated as well.

\section{Governing equations}

The mass and momentum conservation equations governing the motion of fluid can be written as

$$
\frac{\partial \rho}{\partial t}+\frac{\partial}{\partial x_{i}}\left(\rho u_{i}\right)=0
$$




$$
\frac{\partial}{\partial t}\left(\rho u_{i}\right)+\frac{\partial}{\partial x_{j}}\left(\rho u_{i} u_{j}\right)=-\frac{\partial p}{\partial x_{j}}+\frac{\partial \sigma_{i j}}{\partial x_{j}},
$$

where $\rho$ is the fluid density, $u_{i}$ the velocity vector, $p$ the pressure and $\sigma_{i j}$ the viscous stress tensor. Since the velocities in the considered cabin flow and thus the Mach numbers are small, the weakly compressible formulations of equations (1) and (2) are solved. Analyzing the power spectra of the resulting pressure fields at selected locations leads directly to the sound pressure levels in the cabin. The alternative is to solve a wave equation by using an acoustic analogy. Lighthill [7] derived an inhomogeneous wave equation from equations (1) and (2) which is called the Lighthill analogy

$$
\frac{\partial^{2}}{\partial t^{2}}\left(\rho-\rho_{0}\right)-a_{0}^{2} \frac{\partial^{2}}{\partial x_{i}^{2}}\left(\rho-\rho_{0}\right)=\frac{\partial^{2} T_{i j}}{\partial x_{i} \partial x_{j}},
$$

where $\rho_{0}$ denotes the density at rest, $a_{0}$ the speed of sound at rest and $T_{i j}$ is the Lighthill's tensor

$$
T_{i j}=\rho u_{i} u_{j}+\left(\left(p-p_{0}\right)-a_{0}^{2}\left(\rho-\rho_{0}\right)\right) \delta_{i j}-\sigma_{i j} .
$$

In the presence of solid walls, an integral formulation of Lighthill's analogy was first derived by Curle [3] assuming a fixed control surface. Later, Ffowcs Williams and Hawkings [5] as well as Möhring, Müller and Obermeier [9] independently generalized Curle's equation to include bodies with arbitrarily moving boundaries. The integral representations of the sound field in these theories were based on free-space Green's functions including volume integrals as well as surface integrals. The FW-H equation is also an exact rearrangement of the continuity and the momentum equations into the form of an inhomogeneous wave equation. It is based on the free-space Green's function to compute the sound pressure at the receiver position $x$. The FW-H equation for pressure fluctuations radiated into a medium at rest is

$$
\begin{aligned}
& p(\vec{x}, t)-p_{0}=\frac{1}{4 \pi}\left(\frac{\partial}{\partial t} \int_{S}\left[\frac{\left(\rho\left(u_{i}-v_{i}\right)+\rho_{0} v_{i}\right) n_{i}}{r\left(1-M_{r}\right)}\right]_{r e t} d S\right)+ \\
& \frac{1}{4 \pi}\left(\left(-\frac{\partial}{\partial x_{i}}\right) \int_{S}\left[\frac{P_{i j} n_{i}+\rho u_{i}\left(u_{n}-v_{n}\right)}{r\left(1-M_{r}\right)}\right]_{r e t} d S\right)+ \\
& \frac{1}{4 \pi}\left(\frac{\partial^{2}}{\partial x_{i} \partial x_{j}} \int_{V}\left[\frac{T_{i j}}{r\left(1-M_{r}\right)}\right]_{r e t} d V\right)
\end{aligned}
$$

where, $P_{i j}=\left(p-p_{0}\right) \delta_{i j}-\sigma_{i j}, \boldsymbol{r}=\left|\boldsymbol{x}_{\text {receiver }}-\boldsymbol{x}_{\text {surface }}\right|$. Further, $u_{n}$ is the fluid velocity component normal to the surface, $v_{i}$ are the surface velocity components, $v_{n}$ is the 
surface velocity component normal to the surface, $n_{i}$ the surface normal vector, $M_{\mathrm{r}}$ the Mach number of the source towards the receiver. In the case of motionless solid bodies, $M_{r}, v_{i}$ and $v_{n}$ are negligible and the subscript $r e t=t-r / a_{0}$ means that the integral is evaluated at the emission time.

The three sound source terms on the right side of equation (5) represent monopole, dipole and quadrupole sources, respectively. For the low $M a$ number cabin flow and the motionless surface considered here, it turned out that the contributions of monopole and quadrupole sources are negligibly small. Another hybrid approach is to solve the Acoustic Perturbation Equation (APE)

$$
\frac{1}{a^{2}} \frac{\partial^{2}}{\partial t^{2}} p^{a}-\frac{\partial^{2}}{\partial x_{i}^{2}} p^{a}=-\frac{1}{a^{2}} \frac{\partial^{2}}{\partial t^{2}} p^{\prime}
$$

where $p^{a}$ is the acoustic pressure, $p^{\prime}=p-p_{\text {mean }}$ are the pressure fluctuations at the solid walls provided by the flow simulation. Equation (6) does not include a term describing the convection of sound waves since $M a<<1$. The APE is solved with the Rankine-Hugoniot condition $\partial p^{a} / \partial t=-a \nabla p^{a} \cdot n$ at the side boundaries acting as a non-reflecting boundary condition. It must be further noted that both, the FW-H model and the Acoustic Perturbation Equation (APE) are based on pressure perturbations, which are the solution of the weakly compressible Navier-Stokes equations with noslip and impermeability boundary conditions within the CAI and at the cabin walls as well as Neumann boundary conditions for all velocity components and the pressure at the sidewalls.

\section{Computational domain and mesh type}

The flow and the resulting noise generation and propagation are computed in the simplified cabin domain without seats and without passengers, as shown in Fig. 1a). It must be noted that the lateral CAI were modelled in detail as presented in Fig. 1b) in contrast to the ceiling intakes which were simplified by considering only the fins at the outlets.

First, we selected the most suitable meshing strategies provided by StarCCM+, i.e. hexahedral (basic and refined), tetrahedral and polyhedral meshes. Three of the meshes used here had a similar number of vertices for reasons of comparison. The basic hexahedral mesh consisted of 3.8 million cells, 11.2 million faces and 4.1 million vertices, the tetrahedral mesh of 12.5 million cells, 26.7 million faces and 3.7 million vertices and the polyhedral mesh of 1.1 million cells, 5.7 million faces and 4.1 million vertices. Finally, simulations were also performed with a refined hexahedral mesh with 5.1 million cells, 14.5 million faces and 6.4 million vertices. In the hexahedral and tetrahedral mesh cases, the wall boundary layers were resolved with eight parallel layers while only four wall layers were realized in the polyhedral mesh, since more wall layers destabilized the simulation for the latter.

Unsteady simulations were carried out in the LES, DDES and URANS mode. The LES relied on the Wall-Adapting Local-Eddy Viscosity (WALE) subgrid scale model 
[10], the DDES on the DDES-based SST k- $\omega$ turbulence model by Menter [8] and the URANS on the SST k- $\omega$ model as well. In all cases, the filtered weakly compressible Navier-Stokes equations were integrated using second-order time discretization. A second-order central-differencing scheme was used for spatial discretization in URANS, a hybrid second-order up-wind/bounded central-differencing scheme for DDES and a bounded central-differencing scheme for LES. For more details on the discretization schemes, refer to [13].

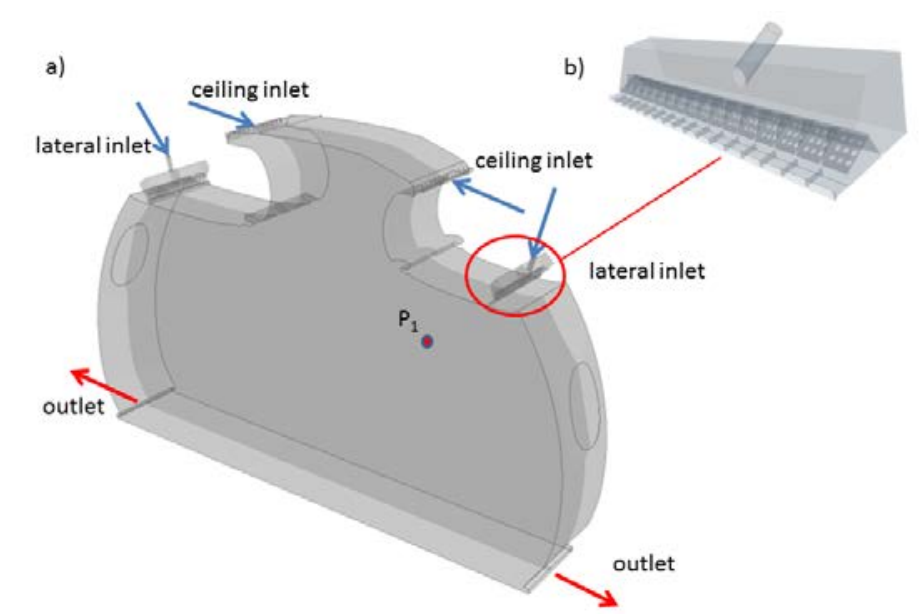

Fig. 1. Computational domain showing the receiver position $\mathrm{P}_{1}$ and the in- and outlets a); and a close-up view of the lateral cabin air intake b).

The simulations were performed with a specified entering mass flow of $0.016 \mathrm{~kg} / \mathrm{s}$ at all inlets. At the outlets, the so-called flow-split outlet conditions were applied. Finally, Neumann boundary (zero normal gradients) conditions were realized at the front and back side of the segment for all velocity components and the pressure whereas no-slip and impermeability conditions were used at all solid walls, i.e. the cabin walls and the solid boundaries within the CAIs. Using Neumann boundary conditions for all flow variables at the side boundaries implies the assumption that the aircraft cabin is infinitely long for the flow which is acceptable for the flow simulations. However, in aero-acoustics, this is a reflective boundary condition which might introduce truncation errors at locations where pressure fluctuations interact with the front and back side boundaries. To study the effect at a specified receiver point $\mathrm{P}_{1}$, a wave equation is solved with a non-reflective boundary condition at the side planes. For the FW-H model, the effect of the side planes is omitted completely, since only noise sources at the cabin walls and within the CAI are considered.

All unsteady simulations were initialized with a steady-state solution and integrated in time with a time step of $\delta t=2.5 \mathrm{e}-5 \mathrm{~s}$ until the total elapsed time reached $t=2.0$ $s$. The sound propagation was computed solving the weakly compressible NavierStokes equation in an LES, DDES or URANS. By storing and evaluating the predicted pressure fields, it is possible to determine the power spectral density (SPL spectrum) based on Fast Fourier Transforms (FFT) of time-series of the static pressure 
fields at selected points or in planes in a post-processing step. SPL is a logarithmic measure of effective sound pressure to a reference value in $d B$ (SPL=10 log $\left.\left(p^{2}{ }_{r m s} / p^{2}{ }_{r e f}\right)\right)$. For air $p_{\text {ref }}=2.0 \mathrm{e}-5 \mathrm{~Pa}$. The main disadvantage is that receiver points have to be selected where the time series of the pressure are stored prior to the start of the simulations or the complete time series of the 3D pressure fields have to be stored. For the present case, nearly $120 \mathrm{~Gb}$ were required to store the time series of the pressure field in the central plane.

Alternatively, time series of the pressure field at the bounding surfaces can be stored. Then, the SPLs at selected receiver points can also be determined solving either the Ffowcs Williams-Hawkings (FW-H) integrals or the APE. The main advantage of the latter approach is the reduced storage requirements.

\section{$4 \quad$ Predicted flow fields and sound propagation}

Contours of the velocity magnitude and pressure fields obtained with URANS, DDES and LES are shown in Figs. 2 - 4. The velocity fields reflect a flow entering the cabin trough the CAI, from where it follows the surface of the luggage compartments until it detaches to form large-scale circulations. Although all simulations predict similar large-scale circulations within the cabin, the predicted pressure fields reflect remarkable quantitative differences. Comparing the URANS, DDES and LES results computed on the same mesh, the LES pressure values are significantly higher than those obtained with DES and URANS. One reason is that LES better captures the generation and interaction of vortices within the CAI, which results in significant pressure losses. This is also important for the sound generation and propagation, since the pressure fluctuations on the CAI's surface are the main sound sources for the complete cabin. Significant acoustic sources also develop on the surfaces of the luggage compartments and the ceiling where the attached flows from the air outlets are attached to the surfaces (see Fig. 2a, 3a and 4a). Therefore, all solid walls were considered as noise source for the FW-H model on the one hand, and for the wave equation on the other hand.

Spectra of the sound pressure level (SPL) (frequency resolution $20 \mathrm{~Hz}$ ) of the DDES obtained for the basic hexahedral, tetrahedral and polyhedral meshes are presented in Fig. 5. The receiver point $\left(\mathrm{P}_{1}\right)$ is located at the position of a passenger's head in a seat next to the aisle, as indicated in Fig. 1. All spectra reveal the same peaks at the frequencies 220, 340, 640 and $1320 \mathrm{~Hz}$. However, the SPL obtained in the computations with the tetrahedral and polyhedral meshes are considerably lower than those computed with the basic hexahedral mesh. For comparison, a microphone measurement in the aisle of the cabin of the test facility Do728 at DLR Göttingen revealed a noise level of $62 \mathrm{~dB}$. This is in reasonable agreement with the maximum in the SPL spectrum predicted with the basic hexahedral mesh. Since the hexahedral mesh performed best, all simulations presented below rely on hexahedral meshes. 

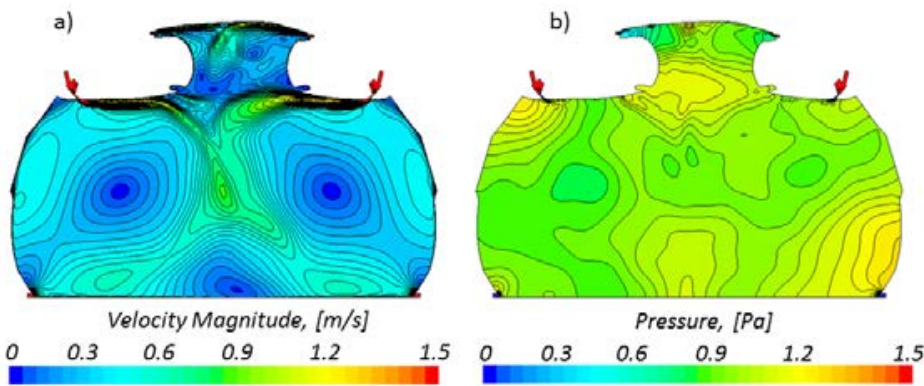

Fig. 2. Snapshots of the distributions of velocity magnitudes (a) and pressure (b) in the central cross-section computed with URANS.
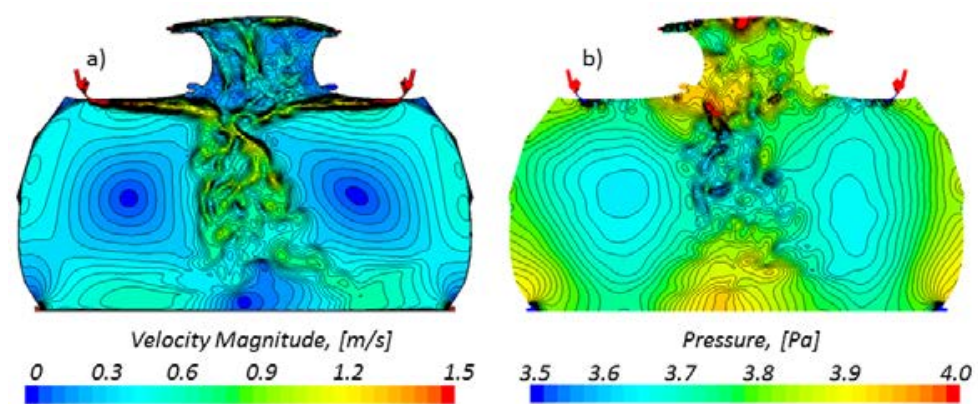

Fig.3. Snapshots of the distributions of velocity magnitudes (a) and pressure (b) in the central cross-section computed with DDES.

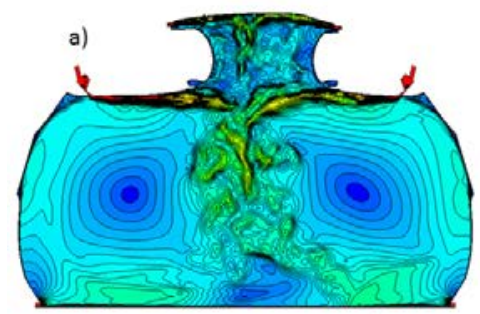

Velocity Magnitude, $[\mathrm{m} / \mathrm{s}]$

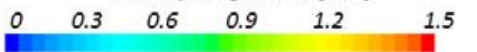

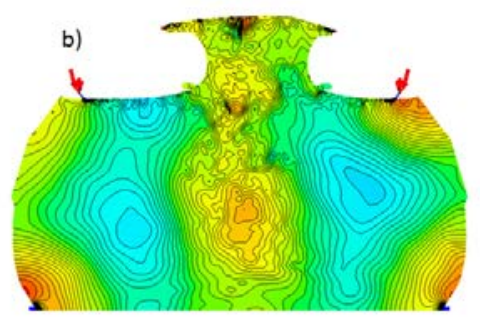

Pressure, $[\mathrm{Pa}]$

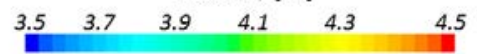

Fig. 4. Snapshots of the distributions of velocity magnitudes (a) and pressure (b) in the central cross-section computed with LES. 

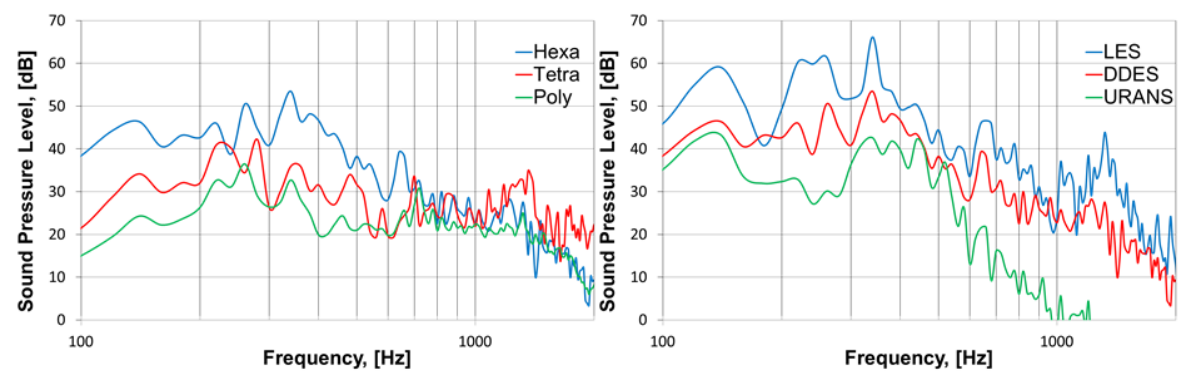

Fig. 5. SPL spectra at receiver point $\mathrm{P}_{1}$ obtained with different meshes using DDES (left) and for LES, DDES and URANS with the basic mesh (right).

The comparison of the SPL spectra at the received point P1 predicted with URANS, DDES and LES shown in Fig. 5(b) further reveals that the SPL obtained with URANS is substantially lower than the LES and DDES spectra. The latter reflect peaks at the same frequencies, i.e. 220, 260, 340, 640 and $1320 \mathrm{~Hz}$ and the low frequency SPLs of the LES are in good agreement with the sound measurements (62 $\mathrm{dB}$ at head level of aisle seat) in the cabin. It must be noted that DDES of cabin air flows do not require less CPU time compared to LES. It is therefore concluded, that LES are recommended for the prediction of sound propagation in the aircraft cabin.

After selecting the most suitable mesh topology and simulation technique, an LES was performed on a refined hexahedral mesh which differs from the basic hexahedral mesh in terms of the more homogeneous cell size distribution. Thus, all results presented below are based on this refined LES.

a)

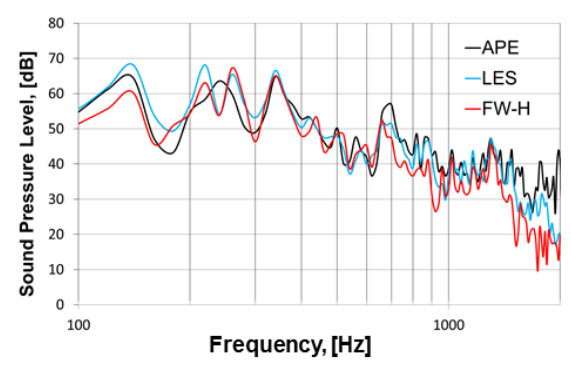

b)

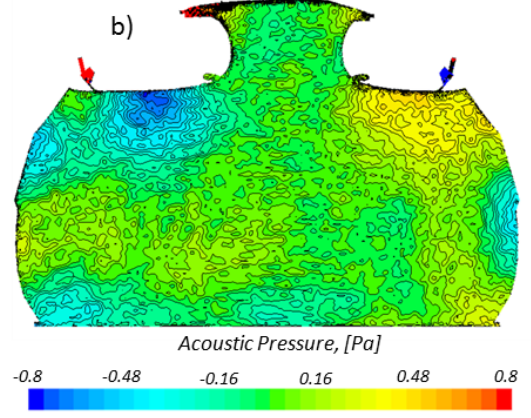

Fig. 6. Comparison of SPL at receiver point $\mathrm{P}_{1}$ obtained by directly analyzing the LES data and solving the FW-H integrals and the wave equation a); acoustic pressure distribution computed with the wave equation APE b).

Contours of the acoustic pressure distribution in the center plane obtained by solving the APE for $0.8 \mathrm{~s}$ are presented in Fig. 6b). Characteristic regions are the vicinity of the in- and outlets and the cabin side walls where the magnitudes of the acoustic 
pressure values reach their maximum. In Fig. 6a), the SPL spectrum obtained directly from the LES pressure fields is compared with spectra resulting from the two investigated hybrid approaches which are based on the FW-H model and the APE, respectively. The three SPL spectra are in good agreement which confirms that the influence of the reflecting boundary condition in the weakly compressible LES is marginal, at least if a receiver point in the center of the cabin is considered.

Finally, in Fig. 7, contours of sound pressure level values of the characteristic frequencies $260 \mathrm{~Hz}$ and $520 \mathrm{~Hz}$ predicted in the cross-section of the Do728 cabin reflect standing waves for the presented frequencies which are obtained solving the weakly compressible Navier-Stoke equations in an LES. Note, that the SPL values at the receiver point correspond to the values directly obtained from LES data (blue line) presented in Fig. 6a) at the selected frequencies.

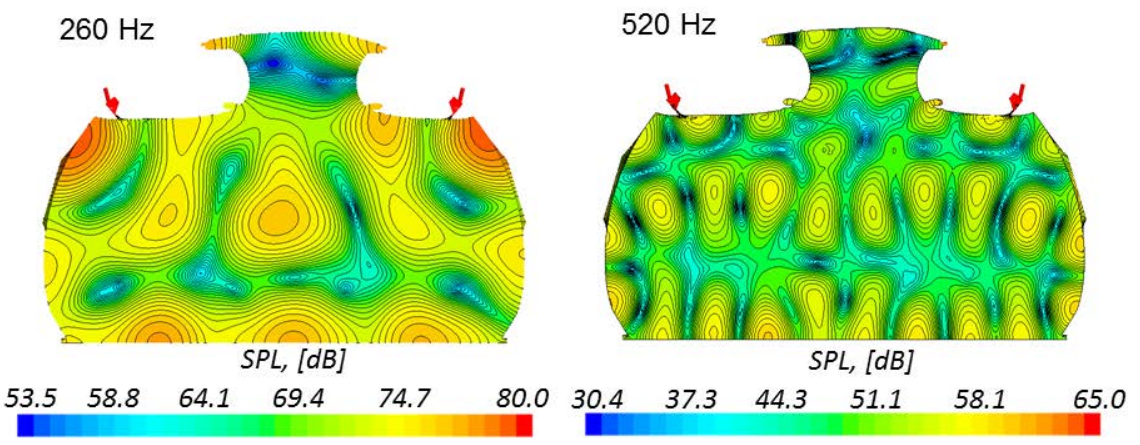

Fig. 7. SPL distribution of two frequencies computed with LES.

\section{$5 \quad$ Conclusions}

Unsteady Reynolds-averaged Navier-Stokes Simulations (URANS), Delayed Detached Eddy Simulations (DDES) and Large Eddy Simulations (LES) of the flow in a segment of an aircraft cabin were performed with the objective to evaluate the applicability of different Computational Aeroacoustics (CAA) approaches for cabin aeroacoustics. A comparison of the results obtained for different meshes and with the above-mentioned techniques revealed that LES solving the weakly compressible Navier-Stokes equation on hexahedral meshes are suited best for future studies of noise propagation in aircraft cabins. Although, the prevailing Neumann boundary conditions at the side boundaries of the cabin segment reflect waves leaving the cabin segment to some extent, no major effect on the predicted SPL at a receiver point in the cabin's center was observed. This was concluded from a comparison of SPLs obtained with two hybrid approaches using the FW-H model and an Acoustic Wave equation (APE), respectively. The present work further demonstrates that it is important to model the cabin walls and their effect on the flow and the wave propagation in the cabin. Thus, the cabin walls, passengers and other obstacles as well as the acoustic characteristics of their surface material will be considered in future simulations. In this respect, also LES of the flow in a complete cabin including seats, pas- 
sengers and other constructive elements will provide a more realistic prediction of SPLs in aircraft cabins.

\section{References}

1. Caro, S., Manera, J., Toppinga, R., Mendonca, F., Validation of a New hybrid CAA Strategy and Application to the Noise Generated by a Flap in Simplified HVAC Duct. AIAA Paper 09-3352, (2009).

2. Cho, M., Kim, H., Oh, C., Ih, K., Khondge, A., Mendonca, F., Lim, J., Choi, E.-S., Ganty, B., Hallez, R., Benchmark Study of Numerical Solvers for Prediction of Interior Noise Transmission excited by A-pillar Vortex, Inter.noise 2014, Melbourne, Australia, (2014).

3. Curle, N., The Influence of Solid Boundaries upon Aerodynamic Sound. Proc. Roy. Soc. (London), A 231, 505-514, (1955).

4. Detry, S., Manera, J., Aero-acoustic Predictions of Industrial Dashboard HVAC Systems. Baltimore, Maryland NOISE-CON 2010, (2010).

5. Ffowcs Williams, J. E., Hawkings, D. L., Sound Generation by Turbulence and Surfaces in Arbitrary Motion. Phil. Trans. Roy. Soc. (London), A 264, 321-342, (1969).

6. Konstantinov, M., Wagner, C., Numerical Simulation of Sound Generation and Propagation in and outside a Cabin Air Intake. Deutscher Luft- und Raum-fahrtkongress 2013, DocumentID: 301330, (2013).

7. Lighthill, M. J., On Sound Generated Aerodynamically. Proc Roy. Soc. (London), A 211, 564-577, (1952).

8. Menter, F. R., Kuntz, M., Adaptation of Eddy Viscosity Turbulence Models to Unsteady Separated Flows behind Vehicles. In Aerodynamics of Heavy Vehicles: Trucks, Buses and Trains, Springer, (2002).

9. Möhring, W., Müller, E.-A., Obermeier, F., Schallerzeugung durch instationäre Strömung als singuläres Störungsproblem, Acustica 21, (1969).

10. Nicoud, F., Ducros, F., Subgrid-Scale Stress Modelling Based on the Square of the Velocity Gradient Tensor. Flow, Turbulence and Combustion, 62, 183-200, (1999).

11. Obermeier, F., Konstantinov, M., Shishkin, A., Wagner, C., Sound Generation by Low Mach Number Flow through Pipes with Diaphragm Orifices. New Results in Numerical and Experimental Mechanics IX. Notes on Numerical Fluid Mechanics and Multidisciplinary Design, Springer, ISSN: 1612-2909.

12. Swertfirm, F., Kreuzinger, J., Peller, N., Hartmann, M., Validation of a Hybrid Simulation Method for Flow Noise Prediction. 18 ${ }^{\text {th }}$ AIAA/CEAS Aeroacoustical Conference, AIAA 2012-2192, (2012).

13. User Guide, Star-CCM+, Version 9.06, CD-adapco, (2014). 